\title{
Towards Pinpointing the Neural Correlates of ACT-R: a Conjunction of Two Model-Based fMRI Analyses
}

\author{
Jelmer P. Borst (jelmer@cmu.edu) \& John R. Anderson (ja@cmu.edu) \\ Dept. of Psychology, Carnegie Mellon University
}

Keywords: model-based fMRI; cognitive modeling; ACT-R.

\section{Introduction}

The aim of this study is to provide a precise mapping of five ACT-R modules on the brain. While there exists a predefined mapping between ACT-R modules and brain regions (e.g., Anderson et al., 2007), these regions are relatively crude (cubes of $\sim 12 \times 12 \times 12 \mathrm{~mm}$ ) and serve in principle only as indicators of module activity. Previously, we have shown that an analysis method called model-based fMRI can provide more detailed whole-brain mappings (Borst, Taatgen, \& Van Rijn, 2011). In the current study, we applied this method to a second dataset and combined the results of both datasets to create an overall mapping.

\section{Method}

In a typical fMRI analysis, the condition structure of the experiment is regressed against the fMRI measurements. This results in brain areas that are active in response to the experimental conditions. In model-based fMRI, predictions of a model are used as a regressor instead, showing brain areas that correlate with activity of model components (e.g., Gläscher \& O'Doherty, 2010).

Recently, we used this method to locate brain areas that correspond to the ACT-R modules in a relatively complex multitask experiment (Borst et al., 2011). We now applied the same method to analyze a dataset that was published by Anderson et al. (2007), who used a more traditional laboratory experiment to show differential brain activity of eight ACT-R modules.

Naturally, the results of both datasets are partly dependent on idiosyncrasies of the respective models and experiments. We therefore subsequently combined the results of the two datasets with a conservative conjunction analysis (Nichols et al., 2005) to create a more stable mapping.

\section{Results}

Figure 1 illustrates the process for the manual module. The left panel shows the results of the model-based analysis of the Anderson et al. (2007) dataset: a region specific to the motor cortex, overlapping with ACT-R's predefined region. The middle panel shows the results of the Borst et al. (2011) dataset: the manual activity of the model correlated most significantly with a region in the visual cortex. In addition, an area in the motor cortex also correlated with the model predictions. The right panel shows the conjunction of both datasets: an area that is constrained to the motor cortex.

Figure 2 shows the results of the conjunction analyses of the other four modules. The aural module correlated with a region in the auditory cortex. The visual module correlated
Manual - Anderson et al., 2007

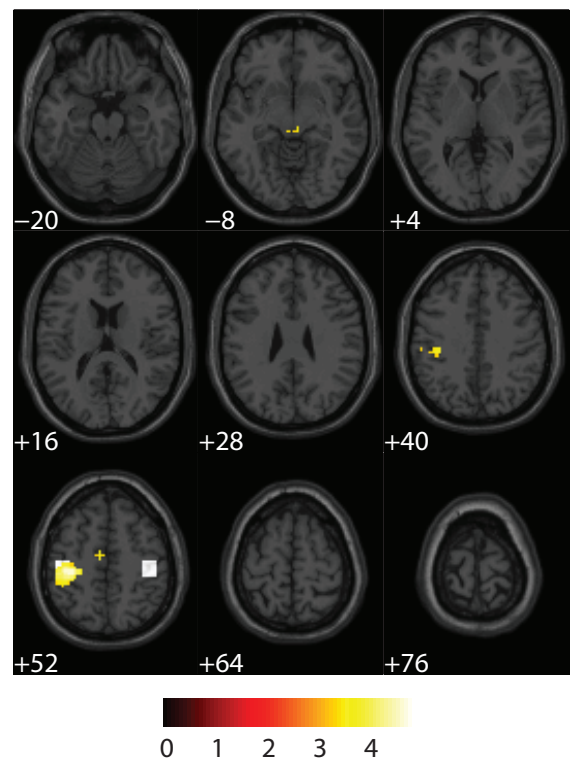

Manual - Borst et al., 2011

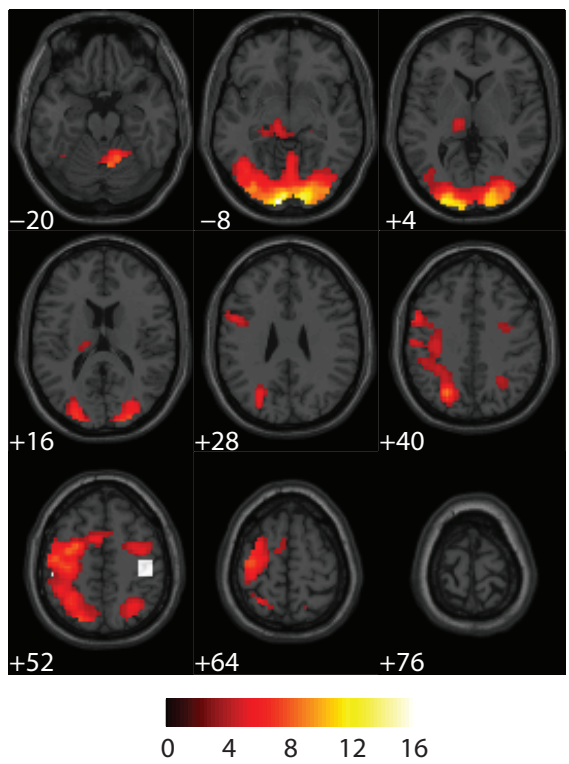

Manual - Conjunction

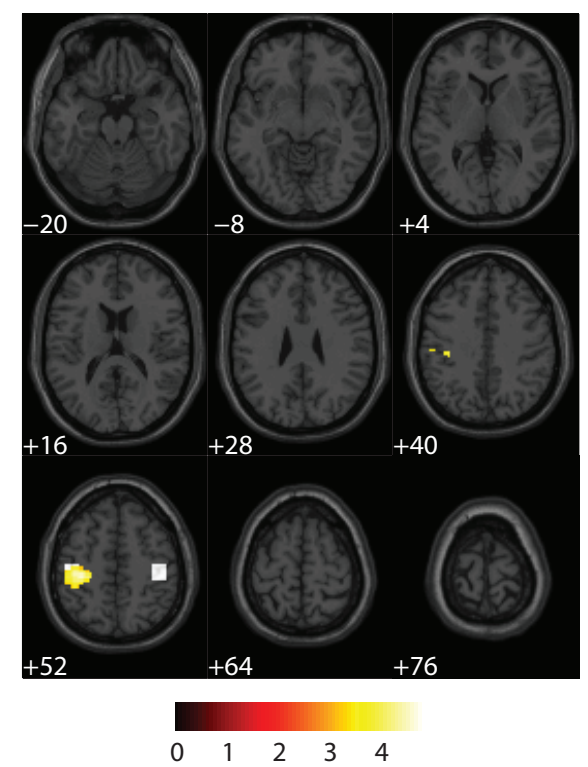

Figure 1. Results for the manual module of the Anderson et al. dataset (left), the Borst et al. dataset (center), and the conjunction (right). All results thresholded at $p<.001$. White squares indicate ACT-R's predefined manual module. 
Aural

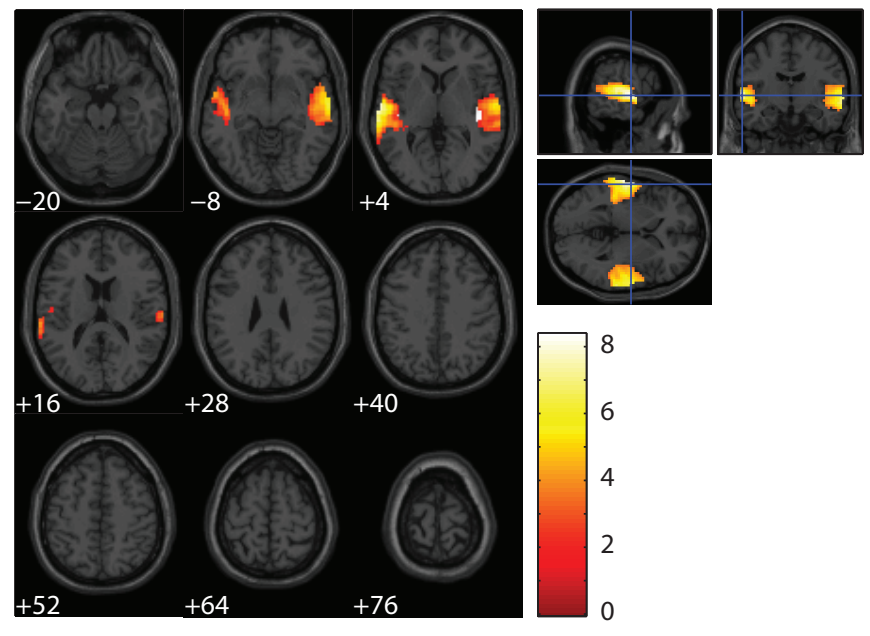

Imaginal

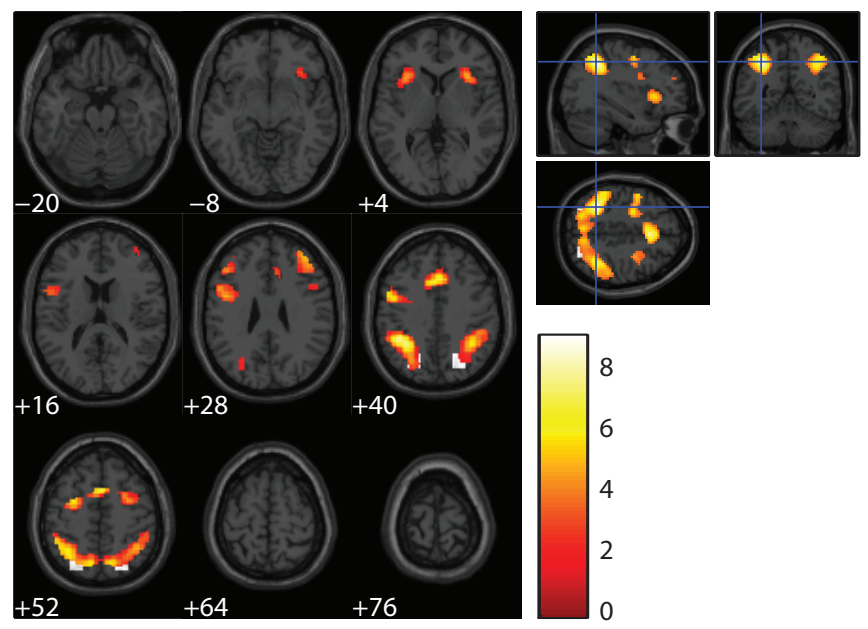

Visual

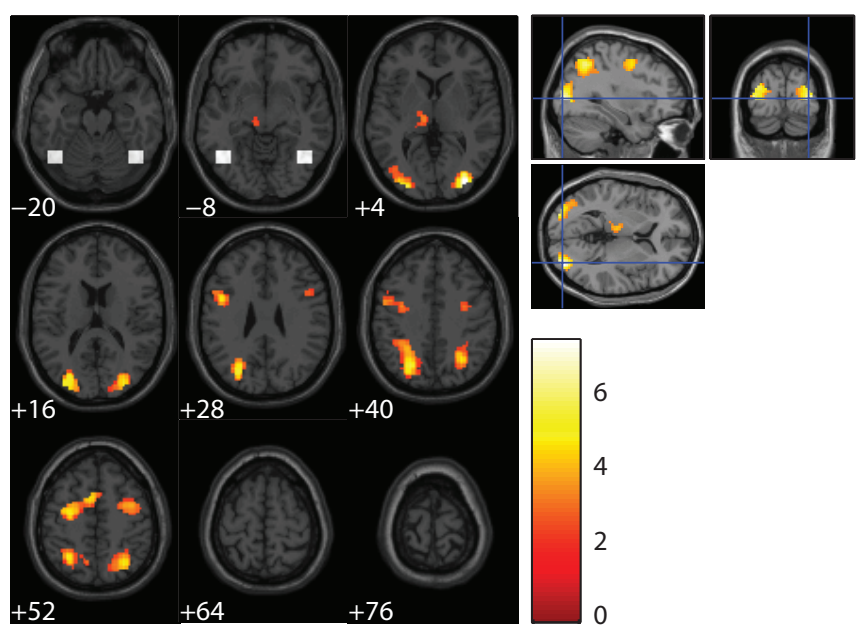

Retrieval

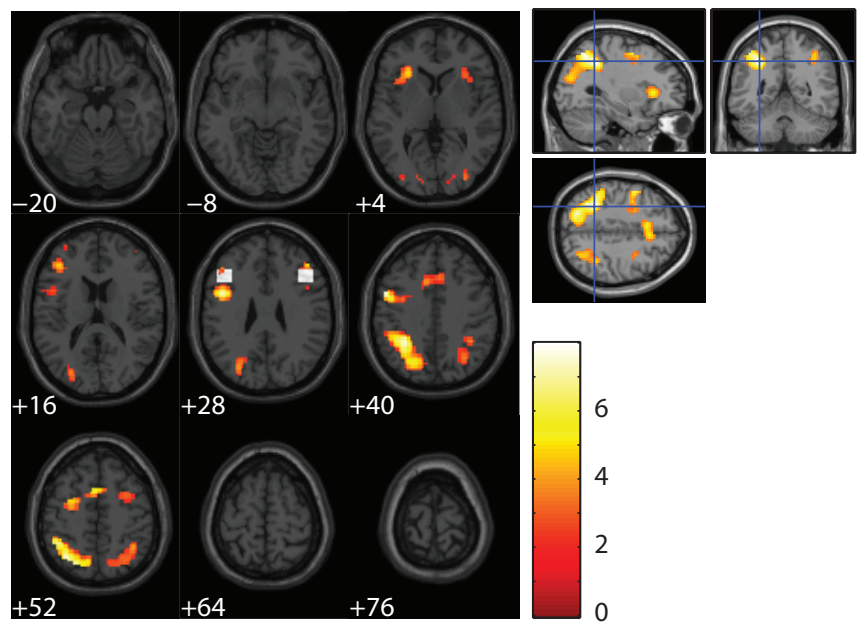

Figure 2. Conjunction results for the aural, visual, retrieval, and imaginal modules, thresholded at $p<.001$. Crosshairs in the ' $3 \mathrm{D}$ ' images indicate the most significant voxel. White squares indicate the respective predefined ACT-R modules.

most with an area in the visual cortex, but also with other areas. As expected, the retrieval module correlated with a prefrontal area, but most strongly with the parietal region that is normally attributed to the imaginal module. Finally, the imaginal module correlated most with the expected area in the parietal cortex, but also with other areas.

\section{Discussion}

Figure 1 highlights the strength of the methodology: while the Borst et al. analysis (2011) resulted in the visual cortex, the combination of the two datasets led to the correct motor region. On the other hand, for the retrieval module we did not find the expected prefrontal region as the best matching area, and for most modules we found activity throughout the brain. While these results might turn out to be the right representations of the modules, it seems to suggest that we should add more datasets to the analysis to create more constrained mappings. In general, combining model-based
fMRI results of multiple experiments seems to be a very promising method to locate the neural correlates of ACT-R.

\section{References}

Anderson, J. R., Qin, Y., Jung, K. J., \& Carter, C. S. (2007). Information-processing modules and their relative modality specificity. Cogn. Psychol., 54(3), 185-217.

Borst, J. P., Taatgen, N. A., \& Van Rijn, H. (2011). Using a symbolic process model as input for model-based fMRI analysis: Locating the neural correlates of problem state replacements. NeuroImage, 58(1), 137-147.

Gläscher, J. P., \& O'Doherty, J. P. (2010). Model-based approaches to neuroimaging: Combining reinforcement learning theory with fMRI data. WIREs Cogn Sci, 1(4), 501-510.

Nichols, T., Brett, M., Andersson, J., Wager, T., \& Poline, J.B. (2005). Valid conjunction inference with the minimum statistic. NeuroImage, 25(3), 653-660. 